\title{
Surface exciton polaritons in individual Au nanoparticles in the far-ultraviolet spectral regime
}

\author{
Ming-Wen Chu, ${ }^{1}$ Cheng-Hsuan Chen, ${ }^{1,2, *}$ F. Javier García de Abajo, ${ }^{3}$ Jin-Pei Deng, ${ }^{4}$ and Chung-Yuan $\mathrm{Mou}^{5}$ \\ ${ }^{1}$ Center for Condensed Matter Sciences, National Taiwan University, Taipei 106, Taiwan \\ ${ }^{2}$ Department of Physics, National Taiwan University, Taipei 106, Taiwan \\ and Institute of Atomic and Molecular Sciences, Academia Sinica, Taipei 106, Taiwan \\ ${ }^{3}$ Instituto de Óptica, CSIC, Serrano 121, 28006 Madrid, Spain \\ ${ }^{4}$ Department of Chemistry, Tamkang University, Taipei County 25137, Taiwan \\ ${ }^{5}$ Department of Chemistry, National Taiwan University, Taipei 106, Taiwan
}

(Received 24 January 2008; published 3 June 2008)

\begin{abstract}
All surface-excitation studies of $\mathrm{Au}$ in the past focused on the well-known $2.4 \mathrm{eV}$ surface plasmon polariton in the visible spectral regime. The existence of surface exciton polaritons is believed to be pristine to the spectral regimes, showing strong excitonic absorptions [F. Yang et al., Phys. Rev. Lett. 64, 559 (1990); Phys. Rev. B 44, 5855 (1991)]. The presence of surface exciton polaritons in far-UV in $\mathrm{Au}(\geq 10 \mathrm{eV})$, where the optical and electronic properties of Au are dominated by broad interband transitions that display characters of rather weak and diffused excitonic oscillator strengths, is not expected and has never been discussed. Reexamining the reports of Yang et al. and using electron energy-loss spectroscopy with a $2 \AA$ electron probe in aloof (optical near-field) setup and real-space energy-filtered imaging, we firmly establish the existence of surface exciton polaritons in individual Au nanoparticles in the far-UV spectral regime. These results indicate that surface exciton polaritons indeed can be excited in weak excitonic onsets in addition to their general believing for the sharp excitonic oscillations. Our experimental observations are further confirmed by the theoretical calculations of electron energy-loss spectra. The unmatched spatial resolution $(2 \AA)$ of the electron spectroscopy technique enables an investigation of individual nanomaterials and their surface excitations in aloof setup. The surface exciton polaritons in individual Au nanoparticles thus represent an example of surface excitations of this type beyond the visible spectral regime and could stimulate further interests in surface exciton polaritons in various materials and applications in novel plasmonics and nanophotonics at high energies via manipulations of the associated surface near fields.
\end{abstract}

DOI: 10.1103/PhysRevB.77.245402

PACS number(s): 79.20.Uv, 78.67.Bf, 73.22.Lp, 73.22.Dj

\section{INTRODUCTION}

Nanoparticles (NPs), a class of emergent materials, serve as a fertile ground to explore unexpected physical characteristics if the property of an individual NP could be faithfully investigated by a spectroscopic technique showing a high spatial resolving capability, such as the exploration of the surface-polariton physics in individual Au NPs here. Surface polaritons are electromagnetic waves that propagate at the surface of materials with the surface wave fields exponentially decaying into the material and bound media (vacuum throughout this work) such that the excitations are localized at the interface and nonradiative in nature. ${ }^{1-5}$ Solving macroscopic Maxwell's equations with proper boundary conditions, the eigenfrequency and the dispersion of surface polaritons can be derived, and the exponential decay of the surface near fields is scaled by $\exp \left[-\alpha_{j}|z|\right]$ with positive and/or negative $z$ for increasing distances from the surface to $\operatorname{vacuum}(j=1) /$ material $(j=2)$ and $\alpha_{j}$ the decay constants normal to the surface $\left[\alpha_{j}=\sqrt{k_{x}^{2}-k_{0}^{2} \varepsilon_{j}}\right.$, where $k_{x}$ is the wave vector parallel to the surface, $\varepsilon_{1}$ is the dielectric constant of vacuum, $\varepsilon_{2}\left(=\varepsilon_{r 2}+i \varepsilon_{i 2}\right)$ is the complex frequency-dependent dielectric function of the isotropic material, and $k_{0}(=\omega / c)$ defines the light line]. ${ }^{1-6}$

Conventional wisdom of nonradiative surface plasmon polaritons (SPPs) requires their existence in the optically metallic and nonabsorbing spectral regime characterized by a negative real part $\left(\left|\varepsilon_{r 2}\right|>\varepsilon_{1}\right)$ and an infinitesimal imaginary part $\left(\varepsilon_{i 2} \ll\left|\varepsilon_{r 2}\right|\right)$ of the dielectric function of the materials so that $k_{x}\left(>k_{0} \sqrt{\varepsilon_{1}}\right)$ is real, and $\alpha_{1}$ and $\alpha_{2}$ are positive numbers. ${ }^{1-3}$ A non-negligible $\varepsilon_{i 2}$ causes strong damping of SPPs (Refs. 7 and 8) and their presence in insulators or the insulating spectral regime of metals are generally not anticipated due to the presence of appreciable $\varepsilon_{i 2}$ and positive $\varepsilon_{r 2}$. For a very thin sample, the surface wave fields from both interfaces start to couple to each other, leading to the splitting of SPPs into symmetric and antisymmetric SPP modes that are distinguished by the respective symmetry of the electric field distributions at the interfaces. ${ }^{1,7,8}$

In distinct contrast to SPPs, the excitations of surface exciton polaritons (SEPs), which are collective oscillations of delocalized excitons at the surface of materials, ${ }^{9-14}$ had been shown to be correlated with sharp transverse excitonic onsets in the materials, $\varepsilon_{i 2} \gg\left|\varepsilon_{r 2}\right| \approx 0.4$.,5 A larger excitonic absorption $\varepsilon_{i 2}$ results in a narrower SEP spectral feature and the sign of $\varepsilon_{r 2}$ does not affect the excitations of SEPs, ${ }^{4-6}$ both at odds with the conventional wisdom of SPPs. ${ }^{1-3}$ Due to the appreciable $\varepsilon_{i 2}$ of SEPs, the derived $k_{x}$ and $\alpha_{1,2}$ are complex and the nonradiative surface-excitation condition is thus accompanied with attenuations along the interfaces and toward the vacuum and the material interior. ${ }^{4,5}$ The symmetric and antisymmetric splittings in SPPs also take place in SEPs as the thickness of the thin-film materials becomes small enough. ${ }^{4,5}$ Compared to the antisymmetric SEP mode, the symmetric mode, which is much weakly attenuated along the interfaces due to its field exclusion from the material interior, 
is known as long-range SEP (LRSEP) with higher excitation probabilities. ${ }^{4,5}$ The antisymmetric mode with lower excitation probabilities is otherwise called short-range SEP (SRSEP). ${ }^{4,5}$ Despite these intriguing characteristics of SEPs, they drew much less attention compared to SPPs in the past, most probably because of the unconventional complex $k_{x}$ and $\alpha_{1,2}$ and the rather counterintuitive condition of $\varepsilon_{i 2} \gg\left|\varepsilon_{r 2}\right|$ $\approx 0$ for their existence. In the presence of a diffused excitonic oscillator strength $\varepsilon_{i 2}>\varepsilon_{r 2}>0$, which is observed in practically all kinds of materials such as semiconductors and insulators above band gaps and the insulating spectral regime of metals, it is, however, not clear whether SEPs could still exist. To exemplify this point is the main goal of this report.

In this work, after Sec. II, first we re-examined the thickness-dependent characteristics of symmetric (LRSEP) and antisymmetric (SRSEP) SEPs reported in Ref. 4 for the sharp excitonic oscillator strengths, $\varepsilon_{i 2} \gg\left|\varepsilon_{r 2}\right| \approx 0$. These derivations were then extended to the case of $\varepsilon_{i 2}>\varepsilon_{r 2}>0$, in which we establish that both symmetric and antisymmetric SEPs, though much broaden, do exist in this condition and have comparable excitation probabilities with weak thickness dependence. In this context, it is no longer necessary to distinguish LRSEP and SRSEP besides their distinct wavefield coupling symmetries. All the details are elucidated in Sec. III. In Sec. IV, we show the existence of SEPs in individual $\mathrm{Au}$ NPs in the far-UV spectral regime, $\geq 10 \mathrm{eV}$, where $\mathrm{Au}$ is insulating $\left(\varepsilon_{r 2}>0\right)$ with its optical and electronic properties dominated by broad transverse interband transitions $\left(\varepsilon_{i 2}>\varepsilon_{r 2}>0\right)$, which play a similar role to the excitonic onsets from deep $5 d$ and hybridized $6 s p$ bands. ${ }^{15,16}$ This is demonstrated experimentally by spatially resolved techniques of scanning transmission electron microscopy (STEM) conjugated to electron energy-loss spectroscopy (EELS). Intriguingly, these SEPs, though quite broad as spectral features, are indeed very well localized at the surface of the NPs and exhibit multipolar characteristics as those demonstrated in SPPs. ${ }^{17-20}$ The multipolar features of SEPs had never been documented, and these experimental results are confirmed by calculations based on the macroscopic dielectric theory for STEM-EELS. ${ }^{17,18}$ It should be noted that all surface-excitation studies of $\mathrm{Au}$ in the past focused on the well-known $2.4 \mathrm{eV}$ SPP in the optically visible, metallic spectral regime. ${ }^{21,22}$ The superb spatial resolution and attainable energy range of STEM-EELS surpass all existing optical techniques and allow a local electronic investigation of any individual objects. ${ }^{23,24}$ Moreover, the geometry of $\mathrm{Au}$ NPs also facilitates the study of surface excitations by electron probe near-field setup. All these favorable factors ultimately lead to these first observations of SEPs in the NPs reported here. Using bulklike $\mathrm{Au}$ films, the broad SEPs would be overwhelmed by the nearby bulk excitations and become indiscernible, and the corresponding conclusion is drawn in Sec. V.

\section{EXPERIMENT}

A systematic STEM-EELS investigation was performed on Au NPs with $\sim 13, \sim 18$, and $\sim 55 \mathrm{~nm}$ in size using a FEI field-emission STEM/TEM, Tecnai F20, operated at $200 \mathrm{kV}$ with a $2 \AA$ electron probe. Throughout the STEM-EELS experiments, a spectrum collection angle of $4.9 \mathrm{mrad}$ was used and the energy resolution was $0.57 \mathrm{eV}$. The real-space spectral imaging, so-called energy-filtered TEM (EFTEM) with a tunable energy-selection slit determining the energy resolution, was conducted on the same microscope.

The synthesis of the Au NPs was reported in a separate work. ${ }^{25}$ The NPs were deposited on $\sim 20$-nm-thick amorphous lacey carbon films coated on a $\mathrm{Cu}$ grid with repeated washings by water and then cyclohexane, followed by proper plasma cleanings to eliminate organic contamination on the NPs prior to spectroscopy investigations.

\section{RE-EXAMINATION OF CONDITIONS FOR SURFACE EXCITON POLARITONS}

The complex frequency-dependent dielectric function of isotropic substances showing an excitonic oscillator strength can be described by

$$
\varepsilon_{2}(\omega)=\varepsilon_{\infty}+\varepsilon_{\infty} \frac{\omega_{P}^{2}-\omega_{T}^{2}}{\omega_{T}^{2}-\omega^{2}+i \omega \gamma},
$$

with $\varepsilon_{\infty}$ as the invariant background dielectric constant, $\omega_{T}$ as the transverse excitonic absorption frequency, $\omega_{P}$ as the longitudinal bulk plasma resonance frequency, and $\gamma$ as the oscillator damping constant. ${ }^{12,13}$ The dielectric function satisfying a sharp excitonic onset as reported in Ref. $4, \varepsilon_{2}=0$ $+i 40$ at $\omega_{T}$ (thus, $\varepsilon_{i 2} \gg\left|\varepsilon_{r 2}\right| \approx 0$ ), is readily given in Fig. 1(a) (inset) by using infinitesimal $\gamma$ and $\omega_{T}=0.5 \omega_{P}$ in Eq. (1), and that of a much broader oscillator strength is shown in Fig. 1(d) (inset) by significantly increasing $\gamma$. To re-examine the thickness $(d)$-dependent characteristics of the symmetric and antisymmetric SEP modes in thin-film materials, as indicated in Ref. 4 for $\varepsilon_{2}=0+i 40$, we analytically derived the respective dispersion equations (2) and (3) with the complex wave vector along the interface as $k_{x}=k_{r}+i k_{i}{ }^{4,5}$

$$
\text { Symmetric mode: } \varepsilon_{2} \alpha_{1}+\varepsilon_{1} \alpha_{2} \tanh \left(\frac{\alpha_{2} d}{2}\right)=0 \text {, }
$$

$$
\text { Antisymmetric mode: } \varepsilon_{2} \alpha_{1}+\varepsilon_{1} \alpha_{2} \operatorname{coth}\left(\frac{\alpha_{2} d}{2}\right)=0 \text {. }
$$

The thus-derived solutions of $k_{r}$ and $k_{i}$ were numerically solved with reference to the wave vector in a very thick material limit $(d \rightarrow \infty), k_{x}=k_{r \infty}+i k_{i \infty}, 4,5$ where the symmetric and antisymmetric modes merge together due to the convergence of the hyperbolic function terms in Eqs. (2) and (3) at large $d$. The numerical results are depicted in Figs. 1(a) and $1(\mathrm{~b})$, and in perfect agreements with those exhibited in Ref. 4 (note the values of $\varepsilon_{1}=1$ and $k_{0}=0.05057 \mathrm{~nm}^{-1}$ used here to shrink the $d$ scale, as compared to Ref. 4).

In Fig. 1(a), the small $k_{r}$ of the antisymmetric mode at $d<10 \mathrm{~nm}, k_{r}<k_{r \infty} \approx k_{0}, 4,5$ indicates that this mode can be directly excited by light, whereas excitations of the symmetric mode at the given $d$ showing $k_{r}>k_{r \infty}$ would require designated optical techniques ${ }^{4-6,11-14}$ or other tools, such as EELS, that provide the extra needed momentum. ${ }^{26}$ At finite $d$ $[<\sim 5 \mathrm{~nm}$, Fig. $1(\mathrm{~b})]$, the vanishing $k_{i}$ of the symmetric 

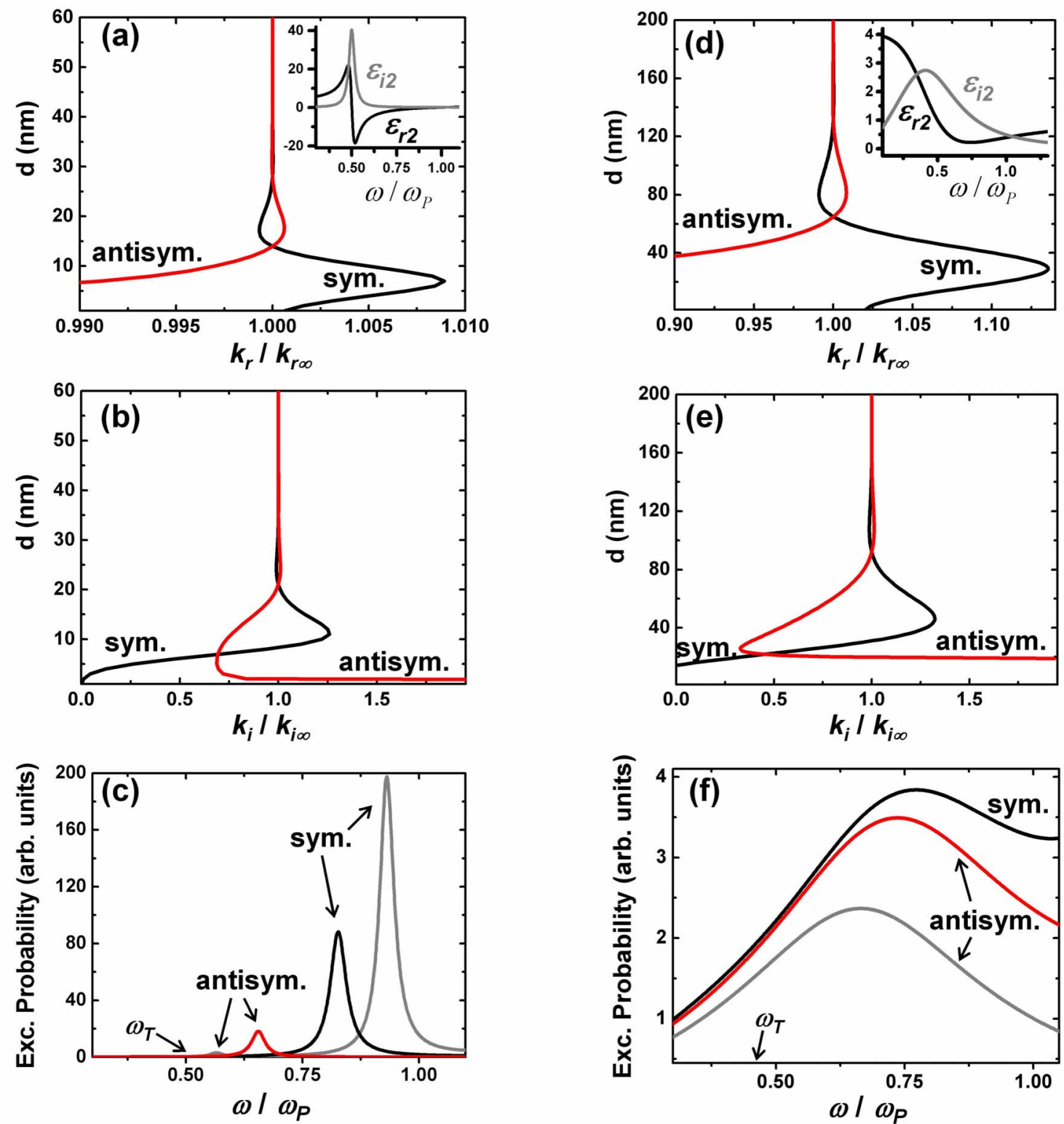

FIG. 1. (Color online) (a) and (b) Thickness (d)-dependent characteristics of $k_{r}$ and $k_{i}$ of the respective symmetric (black) and antisymmetric (red) SEP modes in a thin-film material with reference to $k_{r \infty}$ and $k_{i \infty}$ for the single-interface limit (thick materials). The calculations were performed with $\varepsilon_{2}=0+i 40, \varepsilon_{1}=1$, and $k_{0}=0.05057 \mathrm{~nm}^{-1}$. Inset in (a): the empirical dielectric function of a sharp transverse excitonic oscillator strength signifying $\varepsilon_{2}=0+i 40$. (c) The calculated excitation probabilities of both SEP modes at the thickness of 2 (gray) and $7 \mathrm{~nm}$ (black and red), respectively. (d) and (e) Similar to (a) and (b) with the calculations performed for a rather diffused excitonic excitation, $\varepsilon_{2}=0.62+i 2.19$. Inset in (d): the empirical dielectric function of this diffused excitonic oscillator strength. (f) The calculated excitation probabilities of both SEP modes in (d) and (e) at the thickness of 13 (gray) and $30 \mathrm{~nm}$ (black and red), respectively. Note that the symmetric mode at the thickness of $13 \mathrm{~nm}$ is not shown due to the absence of an apparent spectral feature (see text).

mode further reveals the negligible attenuation along interfaces similar to conventional SPPs, while it is completely the opposite for the antisymmetric mode. The calculations of excitation probabilities by derivations of Eqs. (2) and (3) indeed indicate that the excitation of the symmetric mode at finite $d$ [such as the exaggerated $2 \mathrm{~nm}$, gray curves; Fig. 1(c)] is much more prominent than that of the antisymmetric mode, unambiguously reflecting the suggested LRSEP and SRSEP nature of the respective modes when $d$ is very small. ${ }^{4,5}$ However, $k_{i}$ of both SEP modes quickly become comparable with a slight increase in $d$ [such as at $7 \mathrm{~nm}$, nearly $k_{i}$ crossover; Fig. 1(b)] and a similar change also occurs for their excitation probabilities $[7 \mathrm{~nm}$, black and red curves; Fig. 1(c)]. A clear separation between LRSEP and SRSEP could thus become obscured therein. With nearly identical $k_{i}$, the residual nonequivalent excitation probabilities of both SEP modes [Fig. 1(c)] arise from the more significant attenuations of the antisymmetric SEP along the direction perpendicular to the interface due to the small magnitude of $k_{r}$. In either thin or relatively thicker materials [Fig. 1(c)], it is, however, most important to note that both SEP modes are pertinently located in the gaplike spectral 
range of $\omega_{T}-\omega_{P}$ with $\omega_{T}$ and $\omega_{P}$ as the boundaries. Experimental verifications of the existence of SEPs in such a gaplike regime have been reported for $\mathrm{ZnO},{ }^{11,12} \mathrm{CuBr},{ }^{13}$ and organic semiconducting $\gamma$-cyclo-propyl-bis (1,3,3-trimethylindolenine-2-yl) pentamethinium-tetrafluoroborate (CTIP) ${ }^{14}$ in the visible spectral regime, where all these materials show a sharp oscillator strength with $\varepsilon_{i 2} \gg\left|\varepsilon_{r 2}\right| \approx 0$ as expected. ${ }^{4-6}$ Indeed, it had been documented that onsets of the transverse, single-particle excitonic absorption $\omega_{T}$ could lead to the formation of delocalized Wannier-type excitons in the bulk of materials, readily building up a relevant longitudinal, collective bulk mode at a higher frequency $\omega_{P}{ }^{9,10}$ SEPs that exist in this gapped region are collective surface electromagnetic waves of the delocalized excitons ${ }^{9-14}$ and exhibit mixed transverse longitudinal characters. In contrast to the notable concept of excitonic absorptions in the gaplike $\omega_{T}-\omega_{P}$ range, ${ }^{9-14}$ the SEP excitations, on the other hand, have received much less attention.

Figures 1(d) and 1(e) show the numerical results of $k_{r}$ and $k_{i}$ in a rather diffused excitonic oscillator strength [inset, Fig. 1(d)] with $\gamma$ empirically chosen, which is so large offsetting $\omega_{T}$ from original $0.5 \omega_{P}$ to $0.42 \omega_{P}$. Figures $1(\mathrm{~d})$ and $1(\mathrm{e})$ were obtained with $\varepsilon_{2}=0.62+i 2.19$ (thus, $\varepsilon_{i 2}>\varepsilon_{r 2}>0$ ), $\varepsilon_{1}$ $=1$, and $k_{0}=0.05057 \mathrm{~nm}^{-1}$. These choices of $\gamma, \varepsilon_{2}$, and $k_{0}$ (that of $\sim 10 \mathrm{eV}$ ) have important consequences for realistic material systems, e.g., Au, as will be elaborated later in the text. Indeed, Fig. 1(d) shows similar characteristics to Fig. 1(a) $\left(\varepsilon_{i 2} \gg\left|\varepsilon_{r 2}\right| \approx 0\right)$ with a major difference in the more extended $d$ range of the thickness dependence of $k_{r}$, resulting from the smaller $\operatorname{Re}\left\{\alpha_{2}\right\}$ and $\operatorname{Im}\left\{\alpha_{2}\right\}$ due to the nonzero $\varepsilon_{r 2}$ and smaller $\varepsilon_{i 2}$. The most intriguing features are, however, reflected in the differences between Figs. 1(e) and 1(b). In the case of diffused excitonic onsets [Fig. 1(e)], $k_{i}$ of the symmetric mode is no longer vanishing at small $d(\leq 13 \mathrm{~nm})$ but negative with certain magnitudes. As a function of thickness and compared to Fig. 1(b), the slope-turnover point of $k_{i}$ of the antisymmetric mode $(>13 \mathrm{~nm})$ is now more pronouncedly pushed toward zero and the values of nearby $k_{i}$ are obviously smaller [Fig. 1(e)], suggesting that excitations of the symmetric and antisymmetric SEP modes could be equally favored, if they exist.

Calculations of the excitation probabilities for both symmetric and antisymmetric SEP modes in $\varepsilon_{i 2}>\varepsilon_{r 2}>0[30 \mathrm{~nm}$, black and red curves; Fig. 1(f)] reveal that spectral features, though broad, do appear for the respective modes and locate in the gaplike $\omega_{T}-\omega_{P}$ range, unambiguously showing the existence of SEPs in spectral regimes displaying such diffused excitonic characters [inset, Fig. 1(d)]. Moreover, their excitation probabilities are comparable [30 nm, Fig. 1(f)] with much weaker thickness dependence $[13$ and 30 nm, Fig. 1(f)] with respect to the case of sharp excitonic oscillations [Fig. 1(c)]. In this context, it is, therefore, no longer necessary to define LRSEP and SRSEP besides their distinct wave-field coupling symmetries. Note that no apparent spectral features can be derived for the symmetric mode at $d$ $\leq 13$ nm [e.g., Fig. 1(f), thus not shown] due to its otherwise negative $k_{i}$ that corresponds to an unphysical circumstance with ever-increasing field strength ${ }^{5}$ as it propagates along the interfaces. These characteristics are associated with the smaller $\operatorname{Re}\left\{\alpha_{2}\right\}$ and $\operatorname{Im}\left\{\alpha_{2}\right\}$, which are suggestive of a weaker field exclusion from the material interior ${ }^{5}$ and thus disadvantageous for the build up of the symmetric SEP mode unless for a thick enough film. These features deserve a future separate investigation.

\section{SCANNING TRANSMISSION ELECTRON MICROSCOPY-ELECTRON ENERGY-LOSS SPECTROSCOPY RESULTS AND DISCUSSION}

Having established the excitations of SEPs in diffused oscillator strengths, $\varepsilon_{i 2}>\varepsilon_{r 2}>0$, in Sec. III by numerical calculations of dispersion Eqs. (2) and (3), it is important and desirable to experimentally confirm their existences in realistic materials. This is carried out in the dielectrically isotropic Au NPs using STEM-EELS and energy-filtered electron microscopy. This nanometer-scale sample geometry of Au, instead of thin films, and the high spatial resolution of the spectroscopic techniques are both critical for the experimental observations of SEPs as elucidated hereinafter.

Figure 2(a) is a high-resolution TEM image of the $13 \mathrm{~nm}$ $\mathrm{Au}$ NP along the [110] zone axis (see the corresponding power spectrum), showing the good crystallinity of the NP. All NPs investigated $(\sim 13, \sim 18$, and $\sim 55 \mathrm{~nm})$ are isolated and nearly suspended in vacuum with similar crystallinity and surface cleanness [Fig. 2(a)]. The spherical shape of the NPs was reconfirmed by sample tilting. The clean surface and quasifreestanding configuration are advantageous for studying intrinsic properties of the individual NPs.

We first describe the STEM-EELS spectra [Fig. 2(b)] acquired with the $2 \AA$ electron probe positioned at the center of the individual NPs [scheme, Fig. 2(c); $b \approx 0 \mathrm{~nm} ; b$, impact parameter], which exhibit predominantly bulk excitations, and also at a distance of $1 \mathrm{~nm}$ from the surface of the $13 \mathrm{~nm}$ NP $(b-a=1 \mathrm{~nm} ; a$, radius of NPs), i.e., the aloof or optical near-field setup. ${ }^{17-19}$ With $b \approx 0 \mathrm{~nm}$ for the $55 \mathrm{~nm}$ NP [black curve, Fig. 2(b)], the spectrum features characteristic bulk excitations of $\mathrm{Au}$ at 5.9, 12.0, 16.0, 24.7, and 33.0 $\mathrm{eV}$ and the well-known conventional SPP at $2.4 \mathrm{eV}$ (inset). ${ }^{15,21,22}$ The bulk losses in the spectra of the 13 and 18 nm NPs [green and red curves, Fig. 2(b); $b \approx 0 \mathrm{~nm}$ ] are identical to those observed for the $55 \mathrm{~nm} \mathrm{NP}$, whereas the 2.4-eV SPP is hidden under the rather intense tail (see the arrow) of the zero-loss peak that makes spectral features below $\sim 3 \mathrm{eV}$ difficult to observe 27 except for suitably thick samples with a diminished zero-loss peak, just like in the case of the $55 \mathrm{~nm}$ NP $(b \approx 0 \mathrm{~nm})$. The spectrum of the $13 \mathrm{~nm} \mathrm{NP}$ in near-field setup [blue curve, Fig. 2(b)] exhibits very different features compared to those of bulk excitations in the 13, 18, and 55 $\mathrm{nm}$ NPs $(b \approx 0 \mathrm{~nm})$, notably with pronounced intensities around $10.0 \mathrm{eV}$ and redshifts of the higher energy peaks to 15.0, 23.0, and $31.5 \mathrm{eV}$. The STEM-EELS spectra of the 18 and $55 \mathrm{~nm}$ NPs acquired in near-field setup (1 nm from the NP surface) also show such peak onsets with identical line shapes, widths, and positions to those of the $13 \mathrm{~nm} \mathrm{NP}$ shown in Fig. 2(b) (blue curve). Note that the appearance of noticeable spectral features in STEM-EELS near-field setup must be ascribed to the excitations of nonradiative surface electromagnetic modes or radiative bulk modes. ${ }^{17-19}$

With the $2 \AA$ electron probe passing at a finite distance from the surface of metallic NPs, SPPs are commonly ex- 

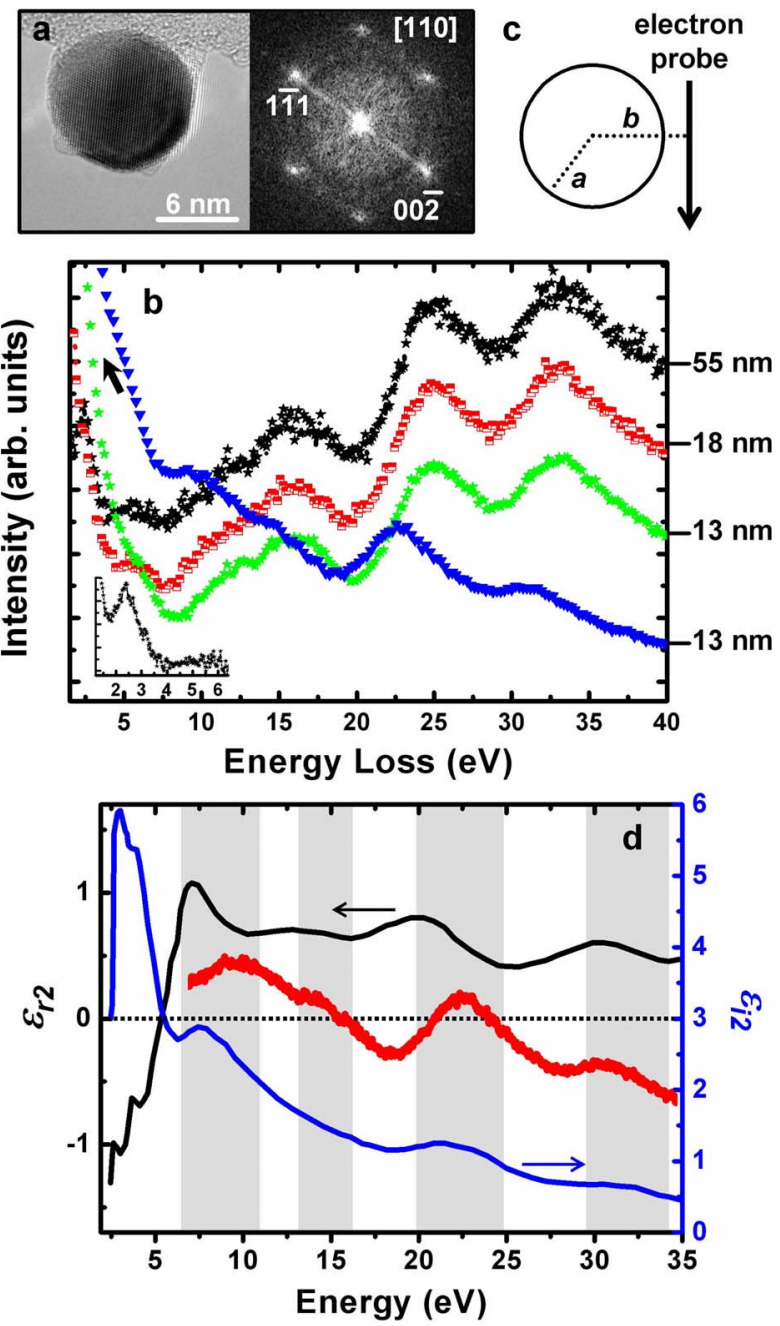

FIG. 2. (Color online) (a) High-resolution TEM image of the 13-nm Au NP with its power spectrum revealing the corresponding [110]-zone axis orientation. (b) STEM-EELS spectra of Au NPs acquired at $b \approx 0$ and $(b-a)=1 \mathrm{~nm}$. The black $(\sim 55 \mathrm{~nm})$, red $(\sim 18 \mathrm{~nm})$, and green $(\sim 13 \mathrm{~nm})$ curves represent spectra recorded at $b \approx 0 \mathrm{~nm}$ for NPs of various sizes. The blue curve was acquired at $(b-a) \approx 1 \mathrm{~nm}$ for the $13 \mathrm{~nm} \mathrm{NP}$. The arrow indicates the intense tail of the zero-loss peak, leading to masking of some spectral features. Inset: an enlarged segment of the spectrum of the $55 \mathrm{~nm} \mathrm{NP}$ with $b \approx 0 \mathrm{~nm}$, showing the presence of the well-known conventional $2.4 \mathrm{eV}$ SPP. All spectra have been vertically shifted and normalized with reference to the peak at $33 \mathrm{eV}$ of the $55 \mathrm{~nm} \mathrm{NP}$ with $b \approx 0 \mathrm{~nm}$ to improve readability. (c) A scheme for the geometry showing $b$ (impact parameter of the electron trajectory with respect to the NP center) and $a$ (NP radius). (d) Real and imaginary parts, $\varepsilon_{r 2}$ and $\varepsilon_{i 2}$, of the dielectric function of bulk Au reproduced from Ref. 15. Red curve: the background-removed spectrum with ( $b$ $-a)=1 \mathrm{~nm}$ in (b), superimposed for highlighting the correspondence between peak onsets and the $\varepsilon_{i 2}>\varepsilon_{r 2}>0$ structures (shadowed regions).

cited because their exponentially decaying near fields into free space can couple to the evanescent field of incident electrons in the vicinity of the NPs. ${ }^{17-19}$ In this near-field setup, Cherenkov radiation (CR) of bulk characteristics in nonabsorbing insulating materials can also be excited due to finite leaking-out probability of the radiation. ${ }^{17,26}$ Examination of the frequency-dependent dielectric function of bulk $\mathrm{Au},{ }^{15}$ Fig. 2(d), reveals that $\varepsilon_{r 2}$ has become positive, thus optically insulating, and is less than one in the far-UV spectral range $(\geq 10.0 \mathrm{eV})$. The positive $\varepsilon_{r 2}$ above $10 \mathrm{eV}$ is obviously at odds with the conventional macroscopic physics for SPPs to occur, negative $\varepsilon_{r 2}$ and infinitesimal $\varepsilon_{i 2},{ }^{1-3}$ and the magnitude of the positive $\varepsilon_{r 2}$ is not even large enough to support CR that requires $\varepsilon_{r 2} \cdot(v / c)^{2}>1$ with $v \sim 0.7 c$ in our experiments at $200 \mathrm{kV}$ (with $v$ as the velocity of incident electrons). ${ }^{17,26}$ The possibility of the peaks observed in nearfield setup [Fig. 2(b)] due to SPPs and CR is completely ruled out.

Further inspection of Fig. 2(d) indicates that the onsets of the peaks at 10.0, 15.0, 23.0, and $31.5 \mathrm{eV}$, Fig. 2(b), are closely correlated with the broad $\varepsilon_{i 2}$ maxima at $\sim 8, \sim 14.5$, $\sim 22$, and $\sim 31 \mathrm{eV}$ and the corresponding oscillating $\varepsilon_{r 2}$ structures (the shadowed regions). These $\varepsilon_{i 2}$ maxima signify the diffused oscillator strengths for bulk transverse interband transitions from deep $5 d$ and $6 s p$ bands, ${ }^{15,16,28}$ thus $\omega_{T}$, and readily build up the respective bulk excitations of longitudinal characters ${ }^{1}$ at $12.0,16.0,24.7$, and $33.0 \mathrm{eV},{ }^{15}$ thus $\omega_{P}$. Further considering the overall condition of $\varepsilon_{i 2}>\varepsilon_{r 2}>0$ discussed above in Sec. III, Figs. 1(d)-1(f), and the dielectric function of $\mathrm{Au}$ at 10.0, 15.0, 23.0, and $31.5 \mathrm{eV}$ [Fig. 2(d)], the existence of these four peaks in the gaplike $\omega_{T}-\omega_{P}$ ranges in Au NPs, therefore, bears strong resemblance to the excitations of SEPs.

Indeed, $\varepsilon_{2}(=0.62+i 2.19)$ used for calculating Figs. 1(d) and $1(\mathrm{e})$ is representative of that in $\mathrm{Au}$ at $\sim 10 \mathrm{eV}\left(k_{0}\right.$ $\sim 0.05057 \mathrm{~nm}^{-1}$ ) [Fig. 2(d)]. The dielectric function empirically derived in Fig. 1(d) (inset) is also representative of that of $\mathrm{Au}$ at $6.5-12 \mathrm{eV}$ [Fig. 2(d)]. The calculated excitation results in Fig. 1(f) thus directly indicate that the peak observed at $10 \mathrm{eV}$ [Fig. 2(b)] arises from the SEP resonance by coupling the near fields of the surface electromagnetic waves $^{4-6}$ to the evanescent field of incident electrons in nearfield setup. The peaks at 15.0, 23.0, and $31.5 \mathrm{eV}$ [Fig. 2(b)] also originate from SEPs since both criteria of $\varepsilon_{i 2}>\varepsilon_{r 2}>0$ and excitations in the gaplike $\omega_{T}-\omega_{P}$ range are equally fulfilled. Although the calculations of Figs. 1(d)-1(f) in Sec. III were performed for materials with a thin-film geometry, ${ }^{4-6}$ the physical essences of surface electromagnetic waves of the SEPs described by $k_{x}$ and $\alpha_{1,2}$ are universal in all geometries such as the perfect applicability of the SEP derivations in thin-film cases, as shown in Figs. 1(a) and 1(b) to a thin islandized silver material. ${ }^{6}$ In $\mathrm{Au}$, the broad interband transitions indicated above should play similar roles to excitons in SEPs.

Figure 3(a) shows the impact-parameter $(b)$ dependent STEM-EELS spectra of the $13 \mathrm{~nm}$ NP in Fig. 2(a) with $(b-a)$ varied from 1 to $10 \mathrm{~nm}$. The peaks at 23.0 and $31.5 \mathrm{eV}(b$ $-a=1 \mathrm{~nm})$ redshift when $b$ increases and those at 10.0 and $15.0 \mathrm{eV}$ show a similar tendency but less obviously due to the noticeable overlap in peak intensity.

It is well established that multipolar Mie surface electromagnetic modes exist at the surface of spherical particles with dipole, quadrupole, hexapole, and higher-order contributions, and the energies of respective multipolar modes increase with the increased order of modes. ${ }^{17-20}$ For increased 

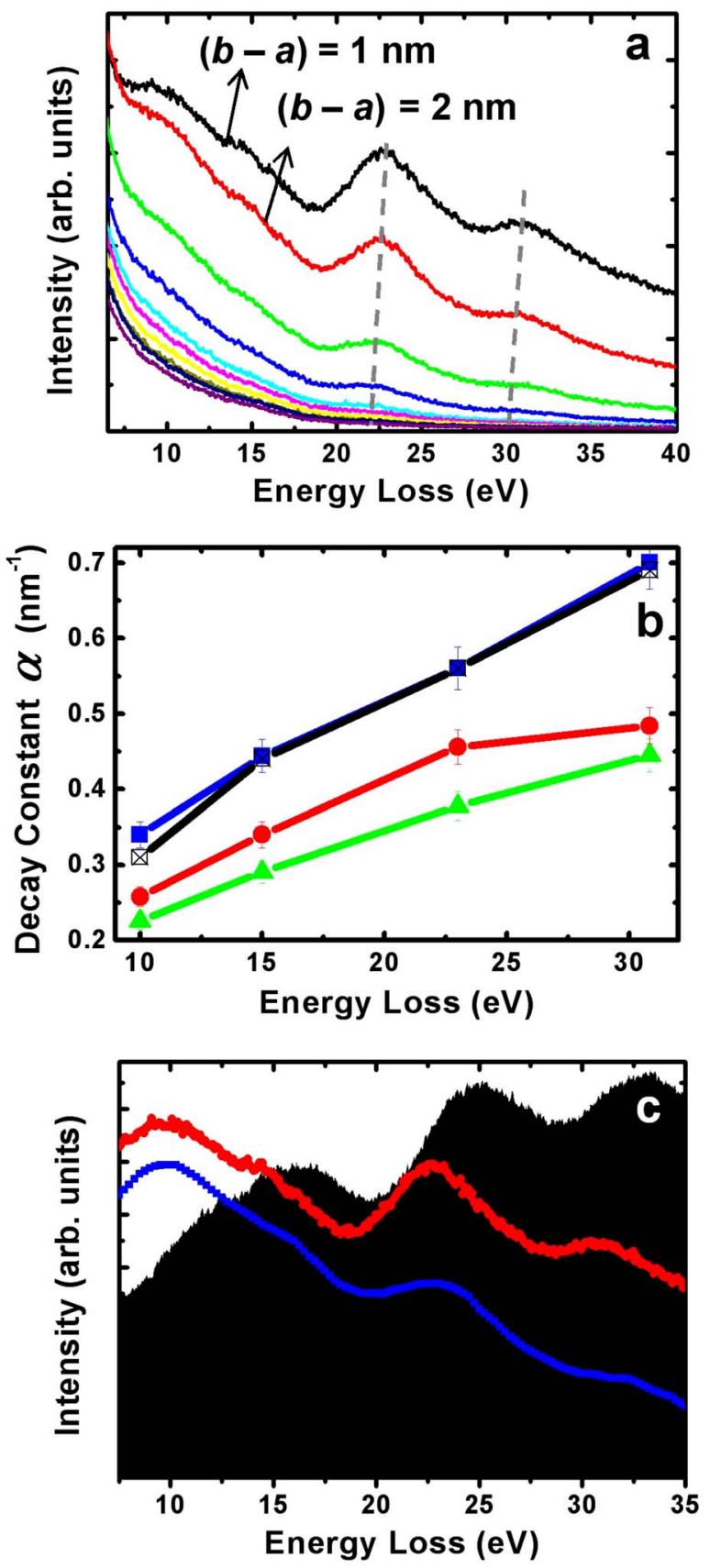

FIG. 3. (Color online) (a) Experimental $b$-dependent STEMEELS spectra of the $13 \mathrm{~nm}$ Au NP shown in Fig. 2(a) with $(b-a)$ varied from 1 to $10 \mathrm{~nm}$ with an interval of $1 \mathrm{~nm}$. The dashed lines are guides for the eyes for redshifts of SEPs with increasing $b$. (b) The blue curve represents the measured decay constants of SEPs shown in (a) for the $13 \mathrm{~nm} \mathrm{NP}$, and the red and green curves are those for the 18 and $55 \mathrm{~nm} \mathrm{NPs}$, respectively. The black curve represents the decay constants derived from calculated $b$-dependent STEM-EELS spectra for a $13 \mathrm{~nm}$ Au NP [e.g., the blue curve in (c)]. (c) Background-removed spectra of the $13 \mathrm{~nm} \mathrm{NP}$ in Fig. 2(b) with $b \approx 0 \mathrm{~nm}$ (black) and $(b-a)=1 \mathrm{~nm}[\mathrm{red}$, identical to the red curve in Fig. 2(d)] compared to the calculated STEM-EELS spectrum for $(b-a)=1 \mathrm{~nm}$ (blue) for a $13 \mathrm{~nm} \mathrm{Au} \mathrm{NP}$. The calculated spectrum has been normalized with reference to the experimental peak at $10 \mathrm{eV}$ of the $13 \mathrm{~nm} \mathrm{NP}$ with $(b-a)=1 \mathrm{~nm}$ and then shifted vertically to improve readability. orders of modes, the number of nodes for the corresponding surface waves increases, leading to a more localized wave field near the surface and faster decay into the free space. ${ }^{17,18}$ The associated near fields for the low-order modes can thus spread to larger distances in vacuum propitious for their excitations at larger $b$, in contrast to the higher-order modes predominant at smaller $b$. The systematic redshifts of $\sim 0.8 \mathrm{eV}$ of the four SEP peaks with increasing $b$ [Fig. 3(a)] are thus suggestive of their respective multipolar nature with contributions from all permitted multipolar modes. Identical $b$-dependent redshifts were also observed for the larger 18 and $55 \mathrm{~nm}$ NPs, revealing that contributions from much higher-order modes supportable by particles with increased sizes $^{17,18}$ are negligible. The characteristic exponential decrease in intensity of the SEPs at 10.0, 15.0, 23.0, and 31.5 $\mathrm{eV}$ [Fig. 3(a)] with increasing $b$ in vacuum was experimentally fitted by a decay function $\exp (-\alpha b)$ characteristic for surface polaritons in general, ${ }^{1-5}$ where $\alpha$ is an empirical decay constant outside the NPs and plays similar role to $\alpha_{1}$ aforementioned regardless of its complex nature in SEPs. ${ }^{4,5}$ Figure 3(b) shows the thus-derived $\alpha$. The monotonic increase in $\alpha$ with the SEP energy is consistent with the kinematical limit, $\omega / v$, for surface polaritons, ${ }^{1,17,18}$ and the greater $\alpha$ for a given peak in the smaller NPs also reflects the characteristic faster decay of the multipolar modes in smaller objects. $^{22}$ An experimental determination of the decay constant inside Au NPs is not plausible as a result of the significant overlap of the SEPs and nearby bulk peaks [Fig. 2(b)].

The existence of SEPs at planar interfaces had been well characterized and the corresponding excitations could be twofold degenerate, symmetric and antisymmetric modes, by the wave-field coupling in thin enough materials. ${ }^{4-6,9-14}$ However, the multipolar surface waves, prone to interfaces with certain curvatures as a result of the corresponding geometrical harmonics, ${ }^{22,23}$ have never been documented in SEPs, though well established for SPPs. ${ }^{17-20,22,23}$ The multipolar nature of the high-energy SEPs in Au NPs, therefore, requires further examinations by theoretical considerations of the STEM-EELS spectra for 13, 18, and $55 \mathrm{~nm} \mathrm{Au} \mathrm{NPs} \mathrm{in}$ the exact experimental condition, $(b-a)=1 \mathrm{~nm}$, on the basis of full solutions of Maxwell's equations using either the boundary element method or an analytical solution taking into account all multipolar expansion of the surface wave fields near the NPs. ${ }^{17,18}$ In the present limit, the difference in calculated results by the two separate methods is indistinguishable. The thus-calculated STEM-EELS spectrum (blue curve) for a $13 \mathrm{~nm} \mathrm{Au} \mathrm{NP,} \mathrm{Fig.} \mathrm{3(c),} \mathrm{is} \mathrm{in} \mathrm{remarkable} \mathrm{agree-}$ ment with the experimental background-removed curve (red). From the calculated $b$-dependent STEM-EELS spectra for a $13 \mathrm{~nm}$ Au NP, we further derived the respective $\alpha$ of the SEPs [black curve, Fig. 3(b)], also in remarkable agreement with the experiments. Such good agreements between calculations and experiments were also found for the 18 and 55 nm Au NPs.

The experimental and theoretical $b$-dependent STEMEELS results for the individual Au NPs in the far-UV spectral regime and the previous numerical calculations of dispersion equations for the condition of $\varepsilon_{i 2}>\varepsilon_{r 2}>0$ in Sec. III have convincingly shown the existence of SEPs with diffused oscillator strengths as intrinsic surface eigenmodes. 

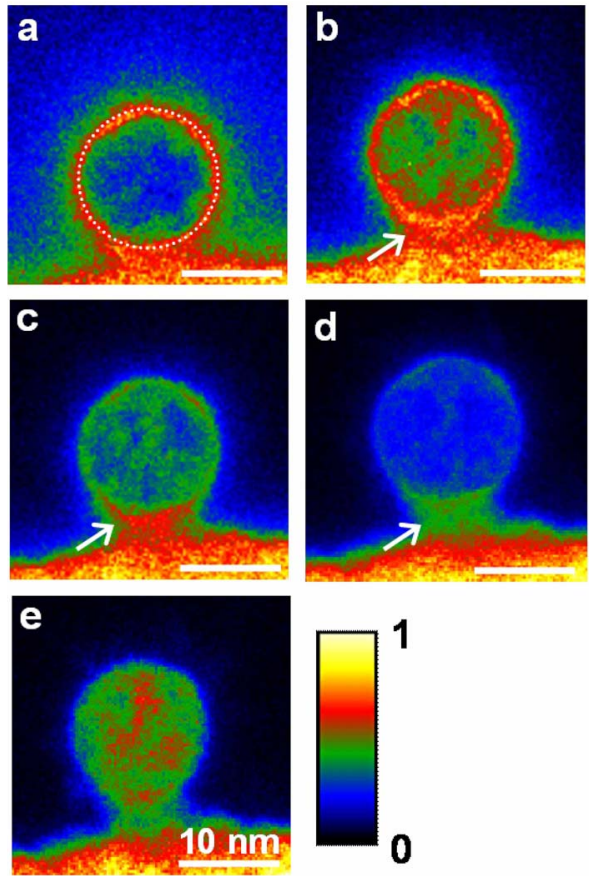

FIG. 4. (Color online) EFTEM imaging of a $13 \mathrm{~nm} \mathrm{Au} \mathrm{NP.}$ [(a)-(e)] EFTEM images acquired with an energy-selection slit of 2 $\mathrm{eV}$ in width at $2-4,10-12,14-16,22-24$, and 32-34 eV, respectively. The dotted circle in (a) indicates the projected surface of the NP. The arrows in (b)-(d) indicate the contrast enhancement due to the coupling to the amorphous carbon supporting film. The linearly normalized contrast scale is shown in the color bar.

The excitations of the corresponding multipolar modes can be mediated by the geometrical constraint. Using a dielectric function similar to that of $\mathrm{Au}$ in the vicinity of $10 \mathrm{eV}$, the absence of an obvious calculated spectral feature for the symmetric mode in a $13 \mathrm{~nm}$ thin film [Fig. 1(f)] does not hinder the current observations because the symmetric mode is eventually a monopole mode (antisymmetric mode, dipolelike) irrelevant to the multipolar nature currently revealed

These SEPs in Au were also investigated by real-space EFTEM imaging of a $13 \mathrm{~nm} \mathrm{NP}$ with the $2 \mathrm{eV}$ energyselection slit at 10-12, 14-16, and 22-24 eV [Figs. 4(b)-4(d)], where the surface peaks at 10.0, 15.0, and 23.0 $\mathrm{eV}$ are located, respectively. The well-known $2.4 \mathrm{eV}$ SPP in $\mathrm{Au}$ was also included for comparison with the energyselection slit at 2-4 eV [Fig. 4(a)]. In the spectral imaging of the $2.4 \mathrm{eV} \mathrm{SPP} \mathrm{[Fig.} \mathrm{4(a)],} \mathrm{the} \mathrm{intensity} \mathrm{maximum} \mathrm{in} \mathrm{the} \mathrm{NP,}$ representing the corresponding spatial location, was found to nicely encircle the circumference, i.e., the surface of the projected image of the NP. This SPP mapping clearly shows the expected localization of the SPP at the NP surface ${ }^{1-5}$ with the evanescent fields decaying both into vacuum and the interior of the NP. The capability of EFTEM in visualizing the SPP in the $13 \mathrm{~nm} \mathrm{NP}$ is remarkable in comparison with the subwavelength spatial resolution of advanced optical near-field techniques, a few tens of nanometers, ${ }^{29-31}$ which is even not adequate for resolving the NP itself. In visualizing SPP by EFTEM or by the complementary STEM-EELS spectral imaging (rastering the finite electron probe on the sample), ${ }^{23,24}$

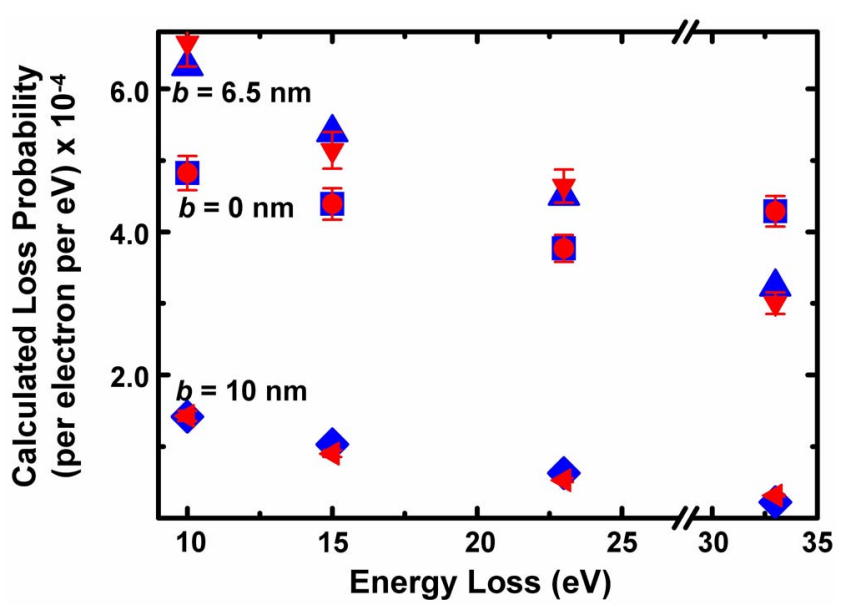

FIG. 5. (Color online) The $b$-dependent STEM-EELS profiles of a $13 \mathrm{~nm}$ NP integrated at given $b$ over the energy windows considered in Figs. 4(b)-4(e). The blue (red) symbols are calculated (experimental) results. At $10,15,23$, and $33 \mathrm{eV}$, the integrated experimental results were normalized to the calculated results for $b$ $=0 \mathrm{~nm}$ to improve the readability.

the delocalized near fields manifested by the contrast decaying in real space [Fig. 4(a)], however, limit the interpretable spatial resolution. ${ }^{23,32}$ Using the spatial resolution criterion of the half spatial maximum at the electronic excitation, ${ }^{23}$ we derived from Fig. 4(a) the resolution of $\sim 6 \mathrm{~nm}$, which represents a pronounced improvement compared to the currently achievable subwavelength optical resolution of a few tens of nanometers. ${ }^{23,29-31}$ This EFTEM resolution also suggests that cautions must be taken when interpreting the spectral imaging of the $2.4 \mathrm{eV}$ SPP in Au NPs well smaller than $\sim 6 \mathrm{~nm}$. In contrast, the sharper near-field decaying inside the NP than into vacuum [Fig. 4(a)] is not an artifact due to the spatial resolution concern, but rather anticipated via the relation of $\alpha_{2}$ as a result of negative $\varepsilon_{r 2}$ in the visible metallic spectral regime in $\mathrm{Au}$ [Fig. 2(d)].

In Figs. 4(b)-4(d), EFTEM reveals intensity maxima at the NP surface similar to those in Fig. 4(a), unambiguously indicating that the broad SEPs at 10.0, 15.0, and $23.0 \mathrm{eV}$ [Fig. 2(b)], though heavily damped in the temporal domain, are indeed very well localized at the NP surface as expected for nonradiative surface electromagnetic waves. ${ }^{1-5}$ In Figs. 4(b)-4(d), the contrast decaying into vacuum also exhibits the qualitatively expected shorter decay length, $\sim v / \omega$, with the increased SEP energy. The spectral imaging at 32-34 eV [Fig. 4(e)], dominated by the bulk loss at $33 \mathrm{eV}$ [Fig. 2(b)], indeed depicts the excitation of bulk nature, and the weak contrast decaying into vacuum is ascribed to the peak overlap with the broad SEP nearby, $31.5 \mathrm{eV}$ [Fig. 2(b)]. In Figs. 4(b)-4(d), the SEP contrasts are particularly intense at the NP-carbon contacts (see the arrows), suggesting strong coupling to the amorphous carbon support. ${ }^{33}$ Figure 5 shows the spectral intensities integrated at the given $b$ in respective Figs. 4(b)-4(e) and those of the calculated $b$-specific STEMEELS spectrum of a $13 \mathrm{~nm}$ NP integrated over the energy windows considered in Figs. 4(b)-4(e) taking into account now not only the surface but also bulk theoretical loss probabilities, exhibiting excellent agreements between experi- 
ments and calculations. The residual nonzero calculated and experimental losses at $33 \mathrm{eV}$ for $b=10 \mathrm{~nm}$ (Fig. 5), observed as contrast decaying into vacuum in Fig. 4(e), are associated with the surface contribution from the nearby $31.5 \mathrm{eV}$ SEP peak.

\section{CONCLUSION}

In summary, the diffused transverse excitonic oscillator strengths with $\varepsilon_{i 2}>\varepsilon_{r 2}>0$ indeed can lead to SEPs, which are collective surface electromagnetic waves exhibiting mixed transverse and longitudinal characters, considering all above experimental evidences and remarkable agreements with theoretical calculations. The existence of SEPs is no longer confined to the sharp excitonic absorption, $\varepsilon_{i 2} \gg\left|\varepsilon_{r 2}\right|$ $\approx 0$, as reckoned previously. ${ }^{4-6}$ An unambiguous experimental demonstration of the presence of SEPs for such diffused excitonic onsets was established in individual Au NPs in the far-UV spectral regime $(\geq 10.0 \mathrm{eV})$, where Au has become optically insulating with its optical and electronic properties dominated by the broad transverse interband transitions $\left(\varepsilon_{i 2}\right.$ $>\varepsilon_{r 2}>0$ ) that play the similar role to excitons. Moreover, the spherical geometry of the Au NPs results in the unexpected multipolar nature of the SEPs, never discussed before. In principle, SEPs observed in the Au NPs as intrinsic surface eigenmodes should also exist at the surface of bulk $\mathrm{Au}$ materials such as films. Unlike the NPs, the STEM-EELS near-field setup in bulk Au materials is much more awkward, limited by the availability of a sample geometry suitable for the aloof electron probe, and the experiments on thin-film samples were thus more easily conducted in transmitting mode. In this later situation, both bulk and surface excita- tions take place simultaneously and the contribution from the bulk losses would, however, overwhelm the much weaker intensity of SEPs nearby [Fig. 2(b)] and obscure their observations. The Au NPs with their geometrical flexibility for the STEM-EELS near-field setup and the spatial resolution ( $2 \AA)$ and energy range of the STEM-EELS technique unparalleled in any optical tools have altogether led to these first observations. We have also calculated the thicknessdependent characteristics of SEPs in the weak excitonic onsets of $\varepsilon_{i 2}>\left|\varepsilon_{r 2}\right|>0$ (negative $\varepsilon_{r 2}$ ), and the results obtained are very similar to those shown in Figs. 1(d)-1(f), suggesting that the sign of $\varepsilon_{r 2}$ does not affect the excitations of SEPs as already known for the sharp excitonic oscillator strengths. ${ }^{4-6}$ Indeed, the surface near fields of the SPP counterparts have given rise to a plethora of fascinating phenomena in the visible spectral regime such as guiding light beyond the subwavelength limit-plasmonics, ${ }^{29-31}$ enhancing Raman scattering cross section by astronomical orders of magnitudes, ${ }^{34,35}$ and molecular imaging and/or engineering. ${ }^{36}$ Our results on SEPs may prompt relevant applications beyond the visible spectral range via manipulations of the associated near fields of SEPs and could stimulate future revival interests in SEPs in various materials with $\varepsilon_{i 2}>\varepsilon_{r 2}>0$.

\section{ACKNOWLEDGMENTS}

This work was supported by the National Science Council of Taiwan under Projects No. NSC94-2120-M-002-016 and No. NSC94-2119-M-002-025. We thank D. Tang of FEI Co. (The Netherlands), V. U. Nazarov and Y. C. Chang at Academia Sinica (Taiwan), and M. Hayashi, J.-K. Wang, and G. Y. Guo at National Taiwan University for fruitful discussion.

\footnotetext{
*Corresponding author; chchen35@ccms.ntu.edu.tw

${ }^{1} \mathrm{H}$. Raether, Excitation of Plasmons and Interband Transitions by Electrons, Springer Tracts in Modern Physics Vol. 88 (SpringerVerlag, Germany, 1980).

${ }^{2}$ R. H. Ritchie, Phys. Rev. 106, 874 (1957).

${ }^{3}$ E. A. Stern and R. A. Ferrell, Phys. Rev. 120, 130 (1960).

${ }^{4}$ F. Yang, J. R. Sambles, and G. W. Bradberry, Phys. Rev. Lett. 64, 559 (1990).

${ }^{5}$ F. Yang, J. R. Sambles, and G. W. Bradberry, Phys. Rev. B 44, 5855 (1991).

${ }^{6}$ F. Yang, G. W. Bradberry, and J. R. Sambles, Phys. Rev. Lett. 66, 2030 (1991).

${ }^{7}$ D. Sarid, Phys. Rev. Lett. 47, 1927 (1981).

${ }^{8}$ F. J. García de Abajo, Rev. Mod. Phys. 79, 1267 (2007).

${ }^{9}$ A. A. Maradudin and D. L. Mills, Phys. Rev. B 7, 2787 (1973).

${ }^{10}$ J. Lagois and B. Fischer, Phys. Rev. B 17, 3814 (1978).

${ }^{11}$ J. Lagois and B. Fischer, Phys. Rev. Lett. 36, 680 (1976).

${ }^{12}$ J. Lagois and B. Fischer, Solid State Commun. 18, 1519 (1976).

${ }^{13}$ I. Hirabayashi, T. Koda, Y. Tokura, J. Murata, and Y. Kaneko, J. Phys. Soc. Jpn. 4, 1215 (1976).

${ }^{14}$ I. Pockrand, A. Brillante, M. R. Philpott, and J. D. Swalen, Opt. Commun. 27, 91 (1978)

${ }^{15}$ M. Schlüter, Z. Phys. 250, 87 (1972).
}

${ }^{16}$ D. A. Papaconstantopoulos, Handbook of the Band Structure of Elemental Solids (Plenum, New York, 1986).

${ }^{17}$ F. J. García de Abajo, Phys. Rev. B 59, 3095 (1999).

${ }^{18}$ F. J. García de Abajo and A. Howie, Phys. Rev. B 65, 115418 (2002).

${ }^{19}$ T. L. Ferrell and P. M. Echenique, Phys. Rev. Lett. 55, 1526 (1985).

${ }^{20}$ N. Yamamoto, K. Araya, and F. J. García de Abajo, Phys. Rev. B 64, 205419 (2001).

${ }^{21}$ B. R. Cooper, H. Ehrenreich, and H. R. Philipp, Phys. Rev. 138, A494 (1965).

${ }^{22}$ I. Romero, J. Aizpurua, G. W. Bryant, and F. J. García de Abajo, Opt. Express 14, 9988 (2006).

${ }^{23}$ J. Nelayah, M. Kociak, O. Stéphan, F. J. García de Abajo, M. Tencé, L. Henrard, D. Taverna, I. Pastoriza-Santos, L. M. LizMarzán, and C. Colliex, Nat. Phys. 3, 348 (2007).

${ }^{24}$ M. Bosman, V. J. Keast, M. Watanabe, A. I. Maaroof, and M. B. Cortie, Nanotechnology 18, 165505 (2007).

${ }^{25}$ J.-P. Deng, W.-C. Shih, and C.-Y. Mou, ChemPhysChem 6, 2021 (2005).

${ }^{26}$ C. H. Chen, J. Silcox, and R. Vincent, Phys. Rev. B 12, 64 (1975).

${ }^{27}$ F. Ouyang, P. E. Batson, and M. Isaacson, Phys. Rev. B 46, 
15421 (1992).

${ }^{28}$ B. R. Cooper, H. Ehrenreich, and H. R. Philipp, Phys. Rev. 138, A494 (1965).

${ }^{29}$ S. A. Maier, M. L. Brongersma, P. G. Kik, S. Meltzer, A. A. G. Requicha, and H. A. Atwater, Adv. Mater. (Weinheim, Ger.) 13, 1501 (2001).

${ }^{30}$ W. L. Barnes, A. Dereux, and T. W. Ebbesen, Nature (London) 424, 824 (2003).

${ }^{31}$ E. Ozbay, Science 311, 189 (2006).
${ }^{32}$ D. A. Muller and J. Silcox, Ultramicroscopy 59, 195 (1995).

${ }^{33}$ A. Rivacoba, N. Zabala, and P. Apell, Surf. Sci. 307-309, 868 (1994).

${ }^{34}$ K. Kneipp, H. Kneipp, I. Itzkan, R. R. Dasari, and M. S. Feld, J. Phys.: Condens. Matter 14, R597 (2002).

${ }^{35}$ H.-H. Wang, C.-Y. Liu, S.-B. Wu, N.-W. Liu, C.-Y. Peng, T.-H. Chan, C.-F. Hsu, J.-K. Wang, and Y.-L. Wang, Adv. Mater. (Weinheim, Ger.) 18, 491 (2006).

${ }^{36}$ P. Schwerdtfeger, Angew. Chem., Int. Ed. 42, 1892 (2003). 